\title{
Model waveform accuracy standards for gravitational wave data analysis
}

\author{
Lee Lindblom, ${ }^{1}$ Benjamin J. Owen, ${ }^{2}$ and Duncan A. Brown ${ }^{3}$ \\ ${ }^{1}$ Theoretical Astrophysics 130-33, California Institute of Technology, Pasadena, California 91125, USA \\ ${ }^{2}$ Institute for Gravitation and the Cosmos, and Center for Gravitational Wave Physics, Department of Physics, The Pennsylvania State \\ University, University Park, Pennsylvania 16802, USA \\ ${ }^{3}$ Department of Physics, Syracuse University, Syracuse, New York 13244, USA
}

(Received 22 September 2008; published 29 December 2008)

\begin{abstract}
Model waveforms are used in gravitational wave data analysis to detect and then to measure the properties of a source by matching the model waveforms to the signal from a detector. This paper derives accuracy standards for model waveforms which are sufficient to ensure that these data analysis applications are capable of extracting the full scientific content of the data, but without demanding excessive accuracy that would place undue burdens on the model waveform simulation community. These accuracy standards are intended primarily for broadband model waveforms produced by numerical simulations, but the standards are quite general and apply equally to such waveforms produced by analytical or hybrid analytical-numerical methods.
\end{abstract}

DOI: 10.1103/PhysRevD.78.124020

PACS numbers: 07.05.Kf, 04.25.D-, 04.25.dg, 04.30.-w

\section{INTRODUCTION}

The purpose of this paper is to derive standards for the accuracy of model waveforms sufficient to ensure that those waveforms are good enough for their intended uses in gravitational wave data analysis. This is a timely and important subject which has received relatively little attention in the scientific literature up to this point. Several gravitational wave detectors [1-3] have now achieved a high enough level of sensitivity that the first astrophysical observations are expected to occur within the next few years. The numerical relativity community has also matured to the point that several groups are now computing model gravitational waveforms for the inspiral and merger of black hole and neutron star binary systems [4-13]. Beyond the pioneering work of Mark Miller [14] and Stephen Fairhurst [15], however, little effort has gone into thinking about the question of how accurate these model waveforms need to be.

This paper contributes to this discussion by formulating a set of accuracy standards for model waveforms sufficient to ensure that those waveforms are able to fulfill the detection and parameter-measurement roles they will be required to play in gravitational wave data analysis. The standards presented here are designed to be optimal in the sense that waveforms of lesser accuracy would result in some loss of scientific information from the data, while more accurate waveforms would merely increase the cost of computing the waveforms without increasing their scientific value in data analysis. Our discussion is done here at a fairly abstract level, with the intention that these accuracy standards should be applicable to model waveforms produced by approximate analytical methods (such as postNewtonian expansions) as well as model waveforms produced by numerical simulations.

Our discussion is divided into two parts: The first part, in Sec. II, assumes that the calibration of the gravitational wave detector is perfect. That is, we assume that the response function used to convert the interferometer output to a gravitational wave signal is known exactly. In this ideal detector case, we present simple derivations for the needed accuracy of model waveforms for detection and separately for parameter measurement purposes. The second part, in Sec. III, evaluates the effects of the calibration error (i.e., the error in the measurement of the response function) on the needed accuracy requirements for model waveforms.

For simplicity, all of our discussion here will be based on the frequency-domain representations of gravitational waveforms $h(f)$, defined as

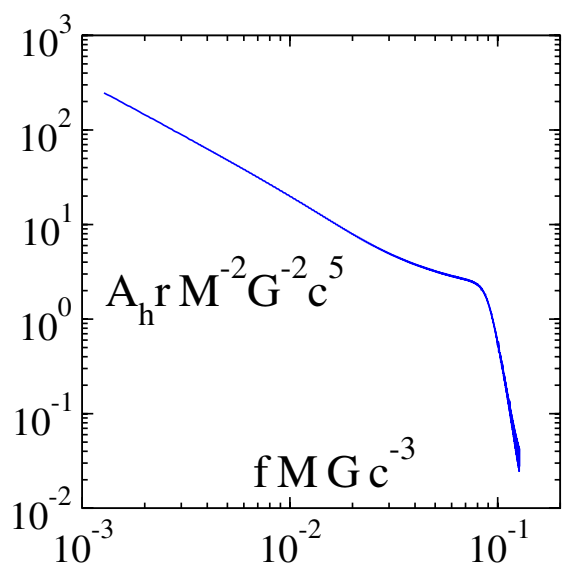

FIG. 1 (color online). Amplitude $A_{h}$ of the frequency-domain gravitational waveform for an equal-mass nonspinning binary black-hole system. Constants representing the total mass, $M$, and the distance, $r$, to the binary system have been introduced to make the graphed quantities dimensionless, independent of the mass of the source, and (asymptotically) independent of the observer's position; $G$ is Newton's constant and $c$ is the speed of light. 


$$
h(f)=\int_{-\infty}^{\infty} h(t) e^{-2 \pi i f t} d t,
$$

where $h(t)$ is the time-domain representation of the waveform. ${ }^{1}$ Since the frequency-domain representation of waveforms is somewhat less familiar to the numerical relativity community, we include Figs. 1 and 2 to illustrate the frequency-domain amplitude $A_{h}$ and phase $\Phi_{h}$, defined as $h(f)=A_{h} e^{i \Phi_{h}}$, of the waveform for a binary black-hole system composed of equal-mass nonspinning holes. The numerical part of this waveform was produced by the Caltech/Cornell numerical relativity group [13], and a post-Newtonian model waveform was stitched on for the lowest part of the frequency range [17]. The constants $M$ and $r$ (used as scale factors in these figures) are, respectively, the total mass and the luminosity distance of the binary system. For clarity of presentation, we have removed the linear in $f$ part of $\Phi_{h}(f)$, which corresponds to shifting the origin of the time coordinate. We have also adjusted the constant part of the phase so that $\Phi_{h}(f)$ does not have a zero and can be graphed more conveniently. These time and phase constants are kinematic parameters, which are not related to the internal dynamics of the waveforms. The resulting $\Phi_{h}(f)$ shown in Fig. 2 is not monotonic in $f$, and this might seem counterintuitive at first. We note that the addition of the linear in $f$ term, $2 \pi t_{0} f$, can make $\Phi_{h}(f)$ either monotonic increasing or decreasing, depending on the value of the time constant $t_{0}$. We also note that the $\Phi_{h}(f)$ illustrated in Fig. 2 is the phase of the Fourier transform of $h(t)$, and consequently has no simple relationship with the phase of the time-domain waveform.

While the accuracy standards derived here apply to any type of gravitational waveform, in practice we expect them to be most useful for broadband waveforms such as those

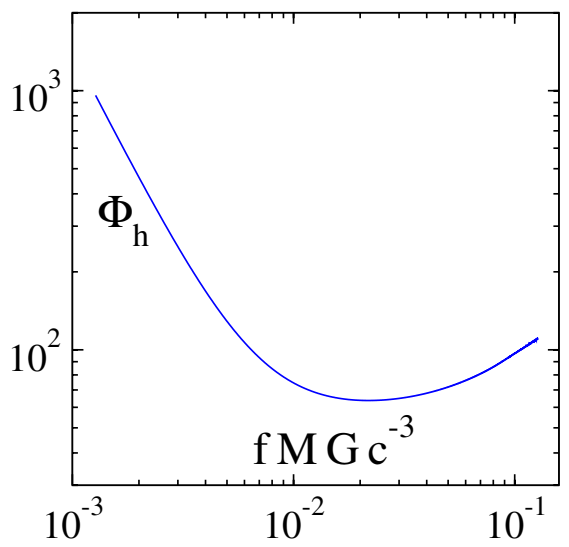

FIG. 2 (color online). Phase $\Phi_{h}$ (measured in radians) of the frequency-domain gravitational waveform for an equal-mass nonspinning binary black-hole system. The constant $M$, representing the total mass of the binary system, has been introduced to express the frequency in dimensionless units, and to make the graphed quantities independent of the mass of the source; $G$ is Newton's constant and $c$ is the speed of light. from compact binary systems. Narrow band gravitational wave signals, such as from rotating neutron stars, are expected to be analyzed using modified versions of the data analysis methods described here, i.e., using the $\mathcal{F}$-statistic matched filter or time-domain heterodyning [18]. The accuracy-standard analysis presented here could easily be extended to these cases $[19,20]$. However narrow band continuous-wave sources have very simple and robust phenomenological waveforms, and so the question of modeling error does not arise in the same way it does for systems with very complex waveforms like compact binary systems.

\section{IDEAL DETECTOR CASE}

We split the discussion of the ideal detector case into three parts. First, we present in Sec. II A a simple derivation of the accuracy of model waveforms needed to ensure no loss of scientific information when the waveform is used to measure the physical properties of a gravitational wave signal. Second, we present in Sec. II B a simple condition on the accuracy of model waveforms needed to ensure a prescribed level of detection efficiency. These accuracy requirements in Secs. II A and II B are optimal in the sense that any model waveform violating them would decrease the scientific effectiveness of the detector. Unfortunately these conditions depend on the detector's noise curve in a complicated way, so enforcing them is somewhat complicated. Therefore we present in Sec. IIC a set of simpler conditions that are nevertheless sufficient to guarantee that the optimal conditions are satisfied. While these sufficient conditions are somewhat stronger than needed, they are much simpler to apply; so we hope they will be easier for the waveform simulation community to adopt and use on a regular basis.

\section{A. Accuracy requirements for measurement}

The question we wish to address here is, how small must the difference between two waveforms be to ensure that measurements with a particular detector are unable to distinguish them? This condition determines how accurate a model waveform $h_{m}$ must be to make it indistinguishable from the exact physical waveform $h_{e}$ through any measurement with a particular detector.

For simplicity, we will perform our analysis in terms of the frequency-domain representation of the waveforms $h_{e}(f)$ and $h_{m}(f)$. Consider the one parameter family of waveforms

\footnotetext{
${ }^{1} \mathrm{We}$ follow the convention of the LIGO Scientific Collaboration [16] (and the signal-processing community) by using the phase factor $e^{-2 \pi i f t}$ in these Fourier transforms; most of the early gravitational wave literature and essentially all other computational physics literature use $e^{2 \pi i f t}$. This choice does not affect any of the subsequent equations in this paper.
} 


$$
h(\lambda, f)=(1-\lambda) h_{e}(f)+\lambda h_{m}(f), \equiv h_{e}(f)+\lambda \delta h(f),
$$

that interpolates between $h_{e}$ and $h_{m}$ as $\lambda$ varies between 0 and 1 . We now ask the related question, how accurately can a particular gravitational wave detector measure this parameter $\lambda$ ? There exists a well developed theory of parameter measurement accuracy for gravitational wave data analysis, discussed, for example, in Finn [21], Finn and Chernoff [22], and Cutler and Flanagan [23]. We construct the noise-weighted inner product $\left\langle h_{e} \mid h_{m}\right\rangle$ given by

$$
\left\langle h_{e} \mid h_{m}\right\rangle=2 \int_{0}^{\infty} \frac{h_{e}^{*}(f) h_{m}(f)+h_{e}(f) h_{m}^{*}(f)}{S_{n}(f)} d f,
$$

where $S_{n}(f)$ is the one-sided power spectral density of the detector strain noise. In defining this inner product, we use the fact that the Fourier transform of the real gravitational wave strain $h(t)$ satisfies $h(f)=h^{*}(-f)$, thus allowing us to define the inner product as an integral over positive frequencies only.

The variance $\sigma_{\lambda}^{2}$ of measurements of the parameter $\lambda$ is given by the expression

$$
\sigma_{\lambda}^{-2}=\left\langle\frac{\partial h}{\partial \lambda} \mid \frac{\partial h}{\partial \lambda}\right\rangle=\langle\delta h \mid \delta h\rangle,
$$

using Eq. (3.20) of Ref. [21], or Eq. (2.8) of Ref. [23]. If the standard deviation $\sigma_{\lambda}$ were greater than one (the parametric distance between $h_{e}$ and $h_{m}$ ), then the two waveforms would be indistinguishable through any measurement with the given detector. Thus the condition, $\sigma_{\lambda}>1$, or equivalently

$$
\langle\delta h \mid \delta h\rangle<1,
$$

ensures that the two waveforms are indistinguishable. ${ }^{2}$ If we consider $h_{e}$ to be the exact waveform, and $\delta h$ to be the difference between the model and the exact waveforms, then the model waveform will be indistinguishable from the exact if and only if $\langle\delta h \mid \delta h\rangle<1$. Our derivation of this condition is based on the simple one-parameter family of waveforms defined in Eq. (2), however, this argument applies to every possible model waveform error $\delta h$. So the argument and resulting condition are completely general: any acceptable waveform model must lie within a ball of unit radius centered on the exact waveform.

We can reexpress this limit, Eq. (5), as a simple condition on the needed phase and amplitude accuracies of the model waveforms. Let $\chi_{e}$ and $\Phi_{e}$ denote real functions representing the logarithmic amplitude and phase of the exact waveform: $h_{e} \equiv e^{\chi_{e}+i \Phi_{e}}$. The model waveform may differ from the exact in both amplitude and phase: $h_{e}+$ $\delta h=h_{e} e^{\delta \chi+i \delta \Phi}$ or to first order $\delta h=(\delta \chi+i \delta \Phi) h_{e}$. It is

\footnotetext{
${ }^{2}$ This inequality, obtained through a different argument, was presented by Stephen Fairhurst in a talk at the "Interplay between Numerical Relativity and Data Analysis" Workshop, at the KITP, UCSB in January 2008.
}

also useful to introduce the normalized waveform $\hat{h}_{e}=$ $h_{e} \rho^{-1}$, which satisfies $\left\langle\hat{h}_{e} \mid \hat{h}_{e}\right\rangle=1$, where $\rho^{2}=\left\langle h_{e} \mid h_{e}\right\rangle$. Using these quantities we can express the inner product $\langle\delta h \mid \delta h\rangle$ in the following way:

$$
\begin{aligned}
\langle\delta h \mid \delta h\rangle & =\rho^{2}\left\langle(\delta \chi+i \delta \Phi) \hat{h}_{e} \mid(\delta \chi+i \delta \Phi) \hat{h}_{e}\right\rangle, \\
& =\rho^{2}\left(\overline{\delta \chi^{2}}+\overline{\delta \Phi^{2}}\right)
\end{aligned}
$$

where the signal-weighted averages of the logarithmic amplitude and phase errors are defined by

$$
\begin{aligned}
& \overline{\delta \chi}^{2} \equiv\left\langle\delta \chi \hat{h}_{e} \mid \delta \chi \hat{h}_{e}\right\rangle, \\
& \overline{\delta \Phi}^{2} \equiv\left\langle\delta \Phi \hat{h}_{e} \mid \delta \Phi \hat{h}_{e}\right\rangle .
\end{aligned}
$$

We can use these definitions to express Eq. (5) as a simple limit on the signal-weighted averages of the logarithmic amplitude and phase errors

$$
\overline{\delta \chi}^{2}+\overline{\delta \Phi}^{2}<\frac{1}{\rho^{2}} .
$$

Equation (5) or, equivalently, Eq. (9) are the basic requirements on model waveforms for measurement purposes. These requirements are optimal in the sense that waveforms more accurate than this would not improve scientific measurements, while less accurate waveforms would degrade some measurements.

\section{B. Accuracy requirements for detection}

The signal-to-noise ratio for the detection of a signal $h_{e}$ using an optimal filter constructed from the model waveform $h_{m}$ is given by

$$
\rho_{m}=\left\langle h_{e} \mid \hat{h}_{m}\right\rangle=\frac{\left\langle h_{e} \mid h_{m}\right\rangle}{\left\langle h_{m} \mid h_{m}\right\rangle^{1 / 2}},
$$

cf. Eq. (A24) of Ref. [23]. The question we wish to address here is, how accurate must the model waveform $h_{m}$ be to ensure no significant loss in the efficiency of detecting the signal $h_{e}$ ? Detections are made when a signal is observed that exceeds a predetermined threshold signal-to-noise ratio. So errors in evaluating the signal-to-noise ratio will decrease the detection efficiency. We must determine therefore how error in the model waveform $h_{m}$ degrades the measured signal-to-noise ratio, $\rho_{m}$, relative to the optimal signal-to-noise ratio $\rho=\left\langle h_{e} \mid h_{e}\right\rangle^{1 / 2}$. We introduce a parameter $\epsilon$, referred to as the mismatch in the gravitational wave data analysis literature [24], that measures this signal-to-noise reduction

$$
\rho_{m}=(1-\epsilon) \rho .
$$

To determine how model waveform error $\delta h$ affects $\epsilon$, we write the model waveform as $h_{m}=h_{e}+\delta h$, and rewrite Eq. (11) in terms of the definitions of $\rho$ and $\rho_{m}$ : 


$$
\frac{\left\langle h_{e} \mid h_{e}+\delta h\right\rangle^{2}}{\left\langle h_{e}+\delta h \mid h_{e}+\delta h\right\rangle}=(1-\epsilon)^{2}\left\langle h_{e} \mid h_{e}\right\rangle .
$$

This equation can be simplified by decomposing $\delta h$ into two parts: $\delta h_{\|}=\hat{h}_{e}\left\langle\delta h \mid \hat{h}_{e}\right\rangle$ and $\delta h_{\perp}=\delta h-\delta h_{\|}$. This $\delta h_{\|}$is proportional to $h_{e}$, while $\delta h_{\perp}$ is orthogonal to it in the sense that $\left\langle\delta h_{\perp} \mid h_{e}\right\rangle=0$. Using these expressions it is straightforward to derive the following relationship between the mismatch $\epsilon$ and the model waveform error

$$
\epsilon=\frac{\left\langle\delta h_{\perp} \mid \delta h_{\perp}\right\rangle}{2\left\langle h_{e} \mid h_{e}\right\rangle} .
$$

We have kept only the lowest-order terms in $\delta h$ (which we assume to be small) in this expression. Equation (13) shows that the mismatch $\epsilon$ is proportional to the square of the distance between two waveforms, as measured by the noise-weighted inner product.

To ensure a high level of detection efficiency while using an optimal filter based on $h_{m}$, we must ensure that the model waveform error $\delta h$ is small enough to prevent $\epsilon$ from becoming unacceptably large. Let $\epsilon_{\max }$ be the maximum mismatch compatible with our target detection efficiency. In that case Eq. (13) places the following limit on the model waveform error:

$$
\left\langle\delta h_{\perp} \mid \delta h_{\perp}\right\rangle<2 \rho^{2} \epsilon_{\max } .
$$

When real searches are conducted to detect signals by matching to a model waveform, the measured signal-tonoise ratio $\rho_{m}$ is maximized over different time and phase offsets of the model waveform. Thus the part of the model waveform phase error linear in $f$ (the part that depends on the time and phase offsets) is not relevant for detection. Strictly speaking then, the inner product that appears in Eq. (14) should be interpreted as the "match" inner product, defined as the Eq. (3) inner product optimized over these time and phase offsets [24].

Equation (14), with $\left\langle\delta h_{\perp} \mid \delta h_{\perp}\right\rangle$ interpreted as the match inner product, gives the optimal condition on the allowed model waveform error for detection purposes. Unfortunately it is not generally possible to determine what the orthogonal part of the waveform error $\delta h_{\perp}$ actually is without knowing the exact waveform, so a simpler, easier to evaluate limit is desirable. We can obtain such a condition by noting that $\langle\delta h \mid \delta h\rangle \geq\left\langle\delta h_{\perp} \mid \delta h_{\perp}\right\rangle$ (where the inner product on the left can be the standard noise-weighted inner product); so a sufficient condition that ensures the target detection efficiency is

$$
\langle\delta h \mid \delta h\rangle<2 \rho^{2} \epsilon_{\max } .
$$

We note that this condition is considerably weaker (depending on the values of $\rho$ and $\epsilon_{\max }$ ) than the limit presented in Eq. (5) to ensure no loss of accuracy in parameter measurements. We can also transform this limit, Eq. (15), into a simple expression for the needed accuracy of the amplitude and phase of model waveforms, in analogy with those found for measurement accuracy in Sec. II A. As before we express the model waveform error in terms of logarithmic amplitude and phase errors: $\delta h=(\delta \chi+$ $i \delta \Phi) h_{e}$. Substituting this into Eq. (15), we arrive at a simple expression for the limit on the signal-weighted averages of the logarithmic amplitude and phase errors required for detection

$$
\overline{\delta \chi}^{2}+\overline{\delta \Phi}^{2}<2 \epsilon_{\max }
$$

The value of the maximum mismatch $\epsilon_{\max }$ that appears in Eqs. (15) and (16) must be set by the demands of the particular data analysis application. Setting $\epsilon_{\max }=0.035$ in a search using a single model waveform template, for example, would result in a reduction in detection rate (for sources that are uniformly distributed in space) of $1-(1-$ $\left(\epsilon_{\max }\right)^{3} \approx 0.10$, a target that is often adopted in LIGO searches for compact binary inspirals [25-28].

Real gravitational wave searches are more complicated, and making the appropriate choice of $\epsilon_{\max }$ is more subtle. Real searches are generally performed by matching discrete template banks of model waveforms with the data. Let $\hat{h}_{b}$ represent one of the (normalized) waveforms in the discrete template bank; let $\hat{h}_{\bar{m}}$ be a (normalized) model waveform whose (appropriately defined) distance from $\hat{h}_{b}$ is the maximum for the given template bank; and let $\hat{h}_{e}$ denote the (normalized) exact waveform whose distance is closest to $\hat{h}_{\bar{m}}$, see Fig. 3 . The model waveform $\hat{h}_{m}$, having the same physical parameters as $\hat{h}_{e}$, may not be identical to $\hat{h}_{\bar{m}}$, since there may be a component of the waveform error tangent to the model waveform submanifold. The quantity $F F=\left\langle\hat{h}_{e} \mid \hat{h}_{\bar{m}}\right\rangle \equiv 1-\epsilon_{\mathrm{FF}}$ is often referred to as the fitting factor [29]; $M M=\left\langle\hat{h}_{\bar{m}} \mid \hat{h}_{b}\right\rangle=1-\epsilon_{\mathrm{MM}}$ is the minimal match [24]; and we refer to $E F F=\left\langle\hat{h}_{e} \mid \hat{h}_{b}\right\rangle=1-\epsilon_{\mathrm{EFF}}$ as the "effective fitting factor." The goal is to have any physical waveform match some model waveform in the template bank with a mismatch that is no greater than the

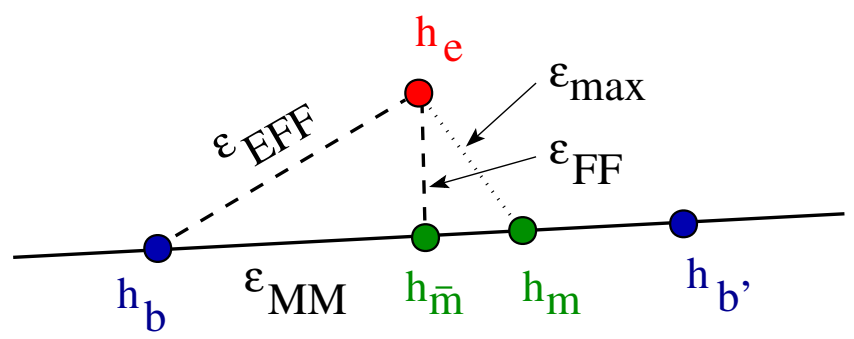

FIG. 3 (color online). The solid line illustrates the model waveform submanifold, with particular members of a discrete template bank $h_{b}$ and $h_{b^{\prime}}$. The model waveform $h_{\bar{m}}$ has the maximum mismatch $\epsilon_{\mathrm{MM}}$ with the template bank waveform $h_{b}$. The closest exact waveform $h_{e}$ has the mismatch $\epsilon_{\mathrm{FF}}$ with $h_{\bar{m}}$ and the total effective mismatch $\epsilon_{\mathrm{EFF}}=\epsilon_{\mathrm{MM}}+\epsilon_{\mathrm{FF}}$ with $h_{b}$. The model waveform $h_{m}$ having the same physical parameters as $h_{e}$ may differ from $h_{\bar{m}}$ due to modeling errors, and its mismatch $\epsilon_{\max }$ will always exceed $\epsilon_{\mathrm{FF}}: \epsilon_{\max } \geq \epsilon_{\mathrm{FF}}$. 
chosen $\epsilon_{\mathrm{EFF}}$ (for example $\epsilon_{\mathrm{EFF}}=0.035$ ). The value of $\epsilon_{\mathrm{FF}}$ is completely determined by the chosen target $\epsilon_{\mathrm{EFF}}$, and the parameter $\epsilon_{\mathrm{MM}}$ which describes the spacing of models in the discrete template bank. Since $\hat{h}_{\bar{m}}$ is the best-fit model waveform for the exact waveform $\hat{h}_{e}$, it follows that the relative waveform vector $\hat{h}_{e}-\hat{h}_{\bar{m}}$ will be orthogonal to any vector tangent to the model waveform submanifold at $\hat{h}_{\bar{m}}$; thus $0=\left\langle\hat{h}_{e}-\hat{h}_{\bar{m}} \mid \hat{h}_{b}-\hat{h}_{\bar{m}}\right\rangle$ in the limit that $\hat{h}_{b}$ and $\hat{h}_{e}$ are infinitesimally close to $\hat{h}_{\bar{m}}$. Writing out this orthogonality condition in terms of the mismatch parameters defined above gives

$$
\epsilon_{\mathrm{FF}}=\epsilon_{\mathrm{EFF}}-\epsilon_{\mathrm{MM}}
$$

a kind of Pythagorean theorem for model waveform mismatches. (Recall from Eq. (13) that the mismatch $\epsilon$ measures the square of the distance between nearby waveforms.) The template banks used for compact binary searches in Initial LIGO are constructed with $\epsilon_{\mathrm{MM}}=0.03$ $[25,26,28]$, implying that the needed accuracy of the model waveforms is $\epsilon_{\mathrm{FF}}=0.005$ when $\epsilon_{\mathrm{EFF}}=0.035$. Since $\hat{h}_{\bar{m}}$ is the closest model waveform to $\hat{h}_{e}$, it follows that the mismatch between $\hat{h}_{e}$ and $\hat{h}_{m}$ (the model waveform with the same physical parameters as $\hat{h}_{e}$ ) will always be greater than the mismatch between $\hat{h}_{e}$ and $\hat{h}_{\bar{m}}$. So it is sufficient to require the maximum mismatch parameter $\epsilon_{\max }$, that appears in Eqs. (15) and (16), to have the maximum value allowed for $\epsilon_{\mathrm{FF}}$. Thus $\epsilon_{\max }$ should have the value 0.005 for the current LIGO searches. It will be appropriate to revisit the issue of optimizing the values of $\epsilon_{\mathrm{MM}}$ and $\epsilon_{\mathrm{FF}}$, including the cost of producing the model waveforms versus the cost of continually filtering with more populous template banks, when fully numerical template banks are constructed in the future.

\section{Sufficient conditions}

The model waveform accuracy requirements for measurement, Eq. (9), and detection, Eq. (16), are optimal in the sense that a model waveform failing to meet these standards will cause a loss of scientific information. Conversely, a model waveform having smaller error than required will result in no added scientific value. Unfortunately these accuracy requirements are somewhat complicated to evaluate, since they place limits on the signal-weighted amplitude and phase errors, $\overline{\delta \chi}$ and $\overline{\delta \Phi}$. These weighted averages must be computed with the frequency-domain waveform $h_{m}(f)$ and the detector noise spectrum $S_{n}(f)$. While model waveforms often scale in a trivial way with the total mass of the gravitational wave source, the detector noise spectrum does not. So the model waveform error must be evaluated separately for each mass, and for each detector noise spectrum. The purpose of this section is to construct a set of simpler-to-apply accuracy requirements that are nevertheless sufficient to guarantee that the optimal conditions, Eqs. (9) and (16), are satisfied. While these sufficient conditions are stricter than needed in many cases, we hope that their ease of use will allow the waveform simulation community to employ them on a regular basis. In this section we present three different sufficient conditions that can be applied to the frequencydomain representations of the waveforms, and one condition that can be applied directly to the time-domain waveforms.

The simplest sufficient conditions can be obtained by

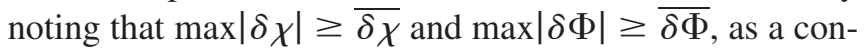
sequence of the definitions of the signal-weighted waveform errors in Eqs. (7) and (8). The following are therefore sufficient conditions which ensure the optimal waveform standard, Eq. (9), is satisfied for measurement

$$
(\max |\delta \chi|)^{2}+(\max |\delta \Phi|)^{2}<\frac{1}{\rho^{2}},
$$

and Eq. (16) is satisfied for detection

$$
(\max |\delta \chi|)^{2}+(\max |\delta \Phi|)^{2}<2 \epsilon_{\max } .
$$

We note that the maxima $\max |\delta \chi|$ and $\max |\delta \Phi|$ in these limits refer to the frequency-domain waveform errors. These requirements are significantly simpler to apply than the optimal waveform standards because they eliminate the detector noise spectrum from the calculation, except for its contribution to the signal-to-noise ratio $\rho$. While simple to evaluate however, these limits on the maxima are stronger than necessary, especially if the amplitude or phase errors are sharply peaked in a narrow range of frequencies or the maximum occurs at a frequency where the amplitude of the wave is very small.

A second, sometimes less demanding sufficient condition on the accuracy of model waveforms can be obtained by noting that $\langle\delta h \mid \delta h\rangle$ can be written as

$$
\begin{aligned}
\langle\delta h \mid \delta h\rangle & =\langle\delta A \mid \delta A\rangle+\left\langle A_{e} \delta \Phi \mid A_{e} \delta \Phi\right\rangle, \\
& \leq\left(\frac{\max |\delta A|}{\bar{n}}\right)^{2}+\left(\frac{\max \left|A_{e} \delta \Phi\right|}{\bar{n}}\right)^{2},
\end{aligned}
$$

where $A_{e}=e^{\chi_{e}}, \delta A=A_{e} \delta \chi$, and the average detector noise $\bar{n}$ is defined as

$$
\frac{1}{\bar{n}^{2}} \equiv 4 \int_{0}^{\infty} \frac{d f}{S_{n}(f)}=\langle 1 \mid 1\rangle .
$$

We can use Eq. (20) to convert Eqs. (5) and (15) into alternate sufficient conditions for model waveform accuracy by introducing a quantity $\bar{C}$,

$$
\bar{C} \equiv \frac{\rho \bar{n}}{A_{e}\left(f_{\mathrm{c}}\right)} .
$$

In this expression $f_{\mathrm{c}}$ is a frequency that characterizes the particular waveform. For the equal-mass binary black-hole waveforms shown in Fig. 1 for example, a convenient choice might be $f_{\mathrm{c}}=0.08 / M$ which occurs near the point in the spectrum where the two black holes merge. This 
quantity $\bar{C}$ is the ratio of the standard signal-to-noise measure $\rho$, and the nonstandard measure $A_{e}\left(f_{\mathrm{c}}\right) / \bar{n}$. Using the definition of $\bar{C}$ and Eq. (20), we can convert Eq. (5) into a simple sufficient condition on the model waveform accuracy for measurement

$$
\left[\frac{\max |\delta A|}{A_{e}\left(f_{\mathrm{c}}\right)}\right]^{2}+\left[\frac{\max \left|A_{e} \delta \Phi\right|}{A_{e}\left(f_{\mathrm{c}}\right)}\right]^{2}<\left(\frac{\bar{C}}{\rho}\right)^{2}
$$

and Eq. (15) into a sufficient condition for detection purposes

$$
\left[\frac{\max |\delta A|}{A_{e}\left(f_{\mathrm{c}}\right)}\right]^{2}+\left[\frac{\max \left|A_{e} \delta \Phi\right|}{A_{e}\left(f_{\mathrm{c}}\right)}\right]^{2}<2 \epsilon_{\max } \bar{C}^{2} .
$$

We note that the errors bounded in Eqs. (18) and (19) are the logarithmic amplitude and phase errors, while those in Eqs. (23) and (24) are errors relative to the fixed characteristic amplitude $A_{e}\left(f_{\mathrm{c}}\right)$. The limits in Eqs. (23) and (24) may therefore be more useful, because they avoid the unnecessarily restrictive conditions on $\max |\delta \chi|$ and $\max |\delta \Phi|$ when those maxima occur at frequencies where the amplitude of the waveform is small.

The requirements in Eqs. (23) and (24) have the disadvantage, however, of involving the quantity $\bar{C}$ which depends on the details of the waveform and the detector noise spectrum. Nevertheless, this quantity can easily be evaluated for a given class of waveforms and a given detector noise spectrum. For example, Fig. 4 illustrates $\bar{C}$ for the case of nonspinning equal-mass binary black-hole waveforms with $f_{\mathrm{c}} \approx 0.08 / M$ using the Initial LIGO noise curve [30] with a $40 \mathrm{~Hz}$ low frequency cutoff [1], and the Advanced LIGO noise curve given by the default parameters of version 1 of the GWINC [31] with a $10 \mathrm{~Hz}$ low frequency cutoff. The scale factor $M_{\odot}$ used in this figure is the mass of the Sun, $M_{\odot}=2.0 \times 10^{33} \mathrm{~g}$, which in

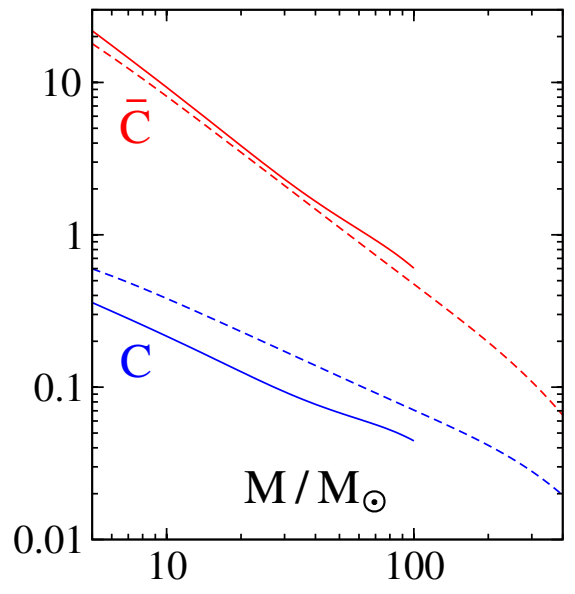

FIG. 4 (color online). Solid curves illustrate $\bar{C}$ and $C$, as defined in Eqs. (22) and (27), as functions of the total mass for nonspinning equal-mass binary black-hole waveforms and the Initial LIGO noise spectrum. Dashed curves give the same quantities computed with the Advanced LIGO noise curve. geometrical time units is $M_{\odot}=4.9 \times 10^{-6} \mathrm{~s}$. This curve shows that $\bar{C} \geq 0.6$ for the mass range of nonspinning binary black-hole systems of primary relevance to Initial LIGO, $5 \leq M / M_{\odot} \leq 100$ [32]. Thus for Initial LIGO it is sufficient to enforce Eqs. (23) and (24) for the case $\bar{C} \approx$ 0.6. The mass range for nonspinning binary black-hole systems of primary relevance for Advanced LIGO extends to $5 \leq M / M_{\odot} \leq 400$, because the low frequency cutoff is $10 \mathrm{~Hz}$ instead of $40 \mathrm{~Hz}$. For Advanced LIGO then, it is appropriate to use the minimum value $\bar{C} \approx 0.06$ when enforcing Eqs. (23) and (24).

A third sufficient condition can be obtained by noting that

$$
\langle\delta h \mid \delta h\rangle=4 \int_{0}^{\infty} \frac{\left|\delta h(f)^{2}\right| d f}{S_{n}(f)} \leq \frac{2\|\delta h(f)\|^{2}}{\min \left[S_{n}(f)\right]},
$$

where $\|\delta h(f)\|$ is the $L^{2}$ norm of $\delta h(f)$, defined as

$$
\|\delta h(f)\|^{2}=2 \int_{0}^{\infty}|\delta h(f)|^{2} d f .
$$

The inequality in Eq. (25) can be converted to sufficient conditions for the optimal waveform requirements by introducing the quantity $C$,

$$
C^{2}=\frac{\rho^{2} \min \left[S_{n}(f)\right]}{2\left\|h_{e}(f)\right\|^{2}},
$$

the ratio of the standard signal-to-noise measure $\rho$ to another nonstandard measure $\left\|h_{e}(f)\right\| / \sqrt{\min S_{n}(f)}$. Using this definition it is straightforward to convert Eq. (25) into sufficient conditions for the optimal error requirements of Eq. (5) for measurement

$$
\frac{\|\delta h(f)\|^{2}}{\left\|h_{e}(f)\right\|^{2}}<\left(\frac{C}{\rho}\right)^{2},
$$

and Eq. (15) for detection

$$
\frac{\|\delta h(f)\|^{2}}{\left\|h_{e}(f)\right\|^{2}}<2 \epsilon_{\max } C^{2} .
$$

As with the previous requirements, these can be written in terms of the amplitude and phase of the frequency-domain waveform: for measurement

$$
\frac{\|\delta A(f)\|^{2}+\left\|A_{e} \delta \Phi(f)\right\|^{2}}{\left\|A_{e}(f)\right\|^{2}}<\left(\frac{C}{\rho}\right)^{2},
$$

and for detection

$$
\frac{\|\delta A(f)\|^{2}+\left\|A_{e} \delta \Phi(f)\right\|^{2}}{\left\|A_{e}(f)\right\|^{2}}<2 \epsilon_{\max } C^{2} .
$$

These requirements depend only on the average value of the model waveform error $\|\delta h(f)\|$, and so they are considerably weaker than (and in this sense are superior to) the conditions in Eqs. (23) and (24). Their biggest drawback is their dependence on the waveform and noise spectrum through the quantity $C$. It is straightforward to show that 
$C \leq 1$ following an argument similar to that which led to Eq. (25). However the exact value of $C$ will depend on the details of the model waveform, including, in particular, the mass of the gravitational wave source. But $C$ can easily be evaluated for a given class of model waveforms and a given detector noise spectrum. For example, Fig. 4 shows a graph of $C$ as a function of the mass for nonspinning equal-mass binary black-hole waveforms (evaluated with the Initial and the Advanced LIGO noise curves). This figure shows that taking $\min (C) \approx 0.02$ in Eqs. (28) and (29) is sufficient for the binary black-hole mass range $5 \leq M / M_{\odot} \leq$ 400 of primary relevance to Advanced LIGO, while taking $\min (C) \approx 0.04$ is sufficient for the mass range $5 \leq$ $M / M_{\odot} \leq 100$ of primary relevance to Initial LIGO [32]. We note that the functions $\bar{C}$ and $C$ illustrated in Fig. 4 apply, strictly speaking, only to the waveform illustrated in Figs. 1 and 2. A more comprehensive investigation will be required to determine how sensitive these functions are to other model waveform parameters (like the mass ratio and the spins of the black holes) and to other effects (like the frequency range of a given model waveform).

Our discussion up to this point has focused on the development of accuracy standards for the frequencydomain representations of model waveforms. This approach simplifies the analysis, and is natural from the LIGO data analysis perspective. However, model waveforms must often be computed in the time domain, e.g., by numerical relativity simulations. While time-domain waveforms can be converted to the frequency domain, doing so is somewhat delicate and requires making judicious choices in performing the needed Fourier transforms-like choosing appropriate windowing functions. Having time-domain versions of the needed accuracy standards would therefore make it much easier for the waveform simulation community to monitor and deliver waveforms of the needed accuracy. The third set of sufficient waveform accuracy requirements, Eqs. (28) and (29), can easily be converted to conditions on the time-domain waveforms. This can be done because the $L^{2}$ norm of a time-domain waveform is identical to the $L^{2}$ norm of its frequency-domain counterpart, e.g., $\|\delta h(t)\|=\|\delta h(f)\|$, by Parseval's theorem. Thus, the following are the corresponding time-domain waveform accuracy standards for measurement:

$$
\frac{\|\delta h(t)\|}{\left\|h_{e}(t)\right\|}<\frac{C}{\rho}
$$

and for detection

$$
\frac{\|\delta h(t)\|}{\left\|h_{e}(t)\right\|}<\sqrt{2 \epsilon_{\max }} C .
$$

It is not generally possible to decompose the real timedomain waveform unambiguously into amplitude and phase. Therefore it is not possible to construct meaningful time-domain analogs of the amplitude and phase limits given in Eqs. (30) and (31).

\section{INCLUDING CALIBRATION ERROR}

In this section we consider the implications of having a detector response function that is not known with absolute precision. First we establish a little notation. Let $v(f)$ denote the direct electronic output of the detector, and $R(f)$ the response function that converts the raw output $v(f)$ into the inferred gravitational wave signal $h(f)$

$$
h(f)=R(f) v(f) .
$$

Let us assume that the measured response function $R(f)$ differs from the correct exact response function $R_{e}(f)$ by $\delta R(f)=R(f)-R_{e}(f)$. This error in the response function will affect measurements in two ways. First, the response of the instrument to a gravitational wave signal $h_{e}$ will produce an electronic output $v_{e}$. Using the measured response function $R$, the signal will be interpreted as the waveform $h=R v_{e}=h_{e} e^{\delta \chi_{R}+i \delta \Phi_{R}}$, where the logarithmic response function amplitude $\delta \chi_{R}$ and phase $\delta \Phi_{R}$ errors are defined by

$$
R=R_{e}+\delta R=R_{e} e^{\delta \chi_{R}+i \delta \Phi_{R}} .
$$

Thus there will be a waveform error

$$
\delta h_{R}=h_{e} e^{\delta \chi_{R}+i \delta \Phi_{R}}-h_{e},
$$

caused by a calibration error in the instrument. The second effect of a calibration error on measurements made with the instrument will be an error in our knowledge of the characteristics of the noise in the detector. In particular the estimated power spectral density of the noise $S_{n}$ will differ from the exact $S_{e}$ due to the calibration error $\delta R$. The estimated power spectral density $S_{n}$ will be related to $S_{e}$ by

$$
S_{n}(f)=S_{e}(f) e^{2 \delta \chi_{R}} .
$$

The idea now is to evaluate the effects of error in the model waveform $h_{m}=h_{e} e^{\delta \chi_{m}+i \delta \Phi_{m}}$, plus the effects of error in the detector response function $R=R_{e} e^{\delta \chi_{R}+i \delta \Phi_{R}}$, on the signal-to-noise ratio of a detected gravitational wave signal

$$
\rho_{m}=\frac{\left\langle h_{e}+\delta h_{R} \mid h_{m}\right\rangle}{\left\langle h_{m} \mid h_{m}\right\rangle^{1 / 2}}
$$

where the inner product is evaluated with respect to the estimated power spectral noise density $S_{n}(f)$ of Eq. (37). The needed calculation is quite long, so we provide a few intermediate steps. Keeping terms through the second order in the two types of error, we find 


$$
\begin{aligned}
\left\langle h_{e}+\delta h_{R} \mid h_{m}\right\rangle= & \rho^{2}+\rho^{2}\left\langle\left(\delta \chi_{m}-\delta \chi_{R}\right) \hat{h}_{e} \mid \hat{h}_{e}\right\rangle \\
& +\frac{\rho^{2}}{2}\left\langle\left(\delta \chi_{m}-\delta \chi_{R}\right) \hat{h}_{e} \mid\left(\delta \chi_{m}-\delta \chi_{R}\right) \hat{h}_{e}\right\rangle \\
& -\frac{\rho^{2}}{2}\left\langle\left(\delta \Phi_{m}-\delta \Phi_{R}\right) \hat{h}_{e} \mid\left(\delta \Phi_{m}-\delta \Phi_{R}\right) \hat{h}_{e}\right\rangle,
\end{aligned}
$$

$$
\begin{aligned}
\left\langle h_{m} \mid h_{m}\right\rangle^{-1 / 2}= & \frac{1}{\rho}-\frac{1}{\rho}\left\langle\left(\delta \chi_{m}-\delta \chi_{R}\right) \hat{h}_{e} \mid \hat{h}_{e}\right\rangle \\
& -\frac{1}{\rho}\left\langle\left(\delta \chi_{m}-\delta \chi_{R}\right) \hat{h}_{e} \mid\left(\delta \chi_{m}-\delta \chi_{R}\right) \hat{h}_{e}\right\rangle \\
& +\frac{3}{2 \rho}\left\langle\left(\delta \chi_{m}-\delta \chi_{R}\right) \hat{h}_{e} \mid \hat{h}_{e}\right\rangle^{2}
\end{aligned}
$$

Combining these using Eq. (38) gives an expression for the effects of model and calibration error on the measured signal-to-noise ratio $\rho_{m},{ }^{3}$

$$
\begin{aligned}
\rho_{m}= & \rho-\frac{\rho}{2}\left\langle\left(\delta \chi_{m}-\delta \chi_{R}\right) \hat{h}_{e} \mid\left(\delta \chi_{m}-\delta \chi_{R}\right) \hat{h}_{e}\right\rangle \\
& -\frac{\rho}{2}\left\langle\left(\delta \Phi_{m}-\delta \Phi_{R}\right) \hat{h}_{e} \mid\left(\delta \Phi_{m}-\delta \Phi_{R}\right) \hat{h}_{e}\right\rangle \\
& +\frac{\rho}{2}\left\langle\left(\delta \chi_{m}-\delta \chi_{R}\right) \hat{h}_{e} \mid \hat{h}_{e}\right\rangle^{2} .
\end{aligned}
$$

It is illuminating to write this expression in the form

$$
\rho_{m}=\rho-\frac{1}{2 \rho}\left\langle\left(\delta h_{m}-\delta h_{R}\right)_{\perp} \mid\left(\delta h_{m}-\delta h_{R}\right)_{\perp}\right\rangle,
$$

where

$$
\left(\delta h_{m}-\delta h_{R}\right)_{\perp}=\delta h_{m}-\delta h_{R}-\hat{h}_{e}\left\langle\delta h_{m}-\delta h_{R} \mid \hat{h}_{e}\right\rangle .
$$

The dependence of the measured signal-to-noise ratio $\rho_{m}$ on the waveform errors, $\delta h_{m}$ and $\delta h_{R}$, can be understood as follows: If (hypothetically) the modeling error were identical to the calibration error, the measured signal and template would still be identical, so the measured matchedfiltering signal-to-noise ratio would be unchanged. If (again hypothetically) the net waveform error $\delta h_{m}-$ $\delta h_{R}$ were proportional to the exact waveform $h_{e}$, the measured signal-to-noise ratio $\rho_{m}$ would be unchanged because such error merely rescales the template which has no effect on $\rho_{m}$. Thus only net waveform error that is orthogonal to $h_{e}$, in the sense of Eq. (43), actually contributes to losses in $\rho_{m}$.

We can use this basic expression, Eq. (42), for the combined effects of the model waveform error and cali-

\footnotetext{
${ }^{3} \mathrm{~A}$ special case of this expression for the signal-to-noise ratio including the first-order calibration-error terms was obtained previously by Bruce Allen [33], and an expression including the second-order calibration-error terms (and some of the firstorder model-error terms) was obtained previously by Sukanta Bose [34].
}

bration error to derive a useful inequality on the signal-tonoise ratio

$$
\begin{aligned}
\rho_{m} & \geq \rho-\frac{1}{2 \rho}\left\langle\delta h_{m}-\delta h_{R} \mid \delta h_{m}-\delta h_{R}\right\rangle \\
& \geq \rho-\frac{1}{2 \rho}\left[\sqrt{\left\langle\delta h_{m} \mid \delta h_{m}\right\rangle}+\sqrt{\left\langle\delta h_{R} \mid \delta h_{R}\right\rangle}\right]^{2} .
\end{aligned}
$$

To aid our understanding of this expression, it is useful to define the ratio of the model waveform error to the response function error, $\eta$,

$$
\left\langle\delta h_{m} \mid \delta h_{m}\right\rangle=\eta^{2}\left\langle\delta h_{R} \mid \delta h_{R}\right\rangle,
$$

which allows us to rewrite Eq. (44) as

$$
\rho_{m} \geq \rho-\frac{1}{2 \rho}(1+\eta)^{2}\left\langle\delta h_{R} \mid \delta h_{R}\right\rangle
$$

If $\eta$ becomes too small, then the error in the measured signal-to-noise ratio $\rho_{m}$ is dominated by the calibration error, and further reductions in the model waveform error have little effect on our ability to make measurements or detections. The idea then is to place a limit on $\eta$ which ensures that the model waveform is only as accurate as it needs to be to achieve the ideal-detector accuracy standards of Sec. II. It is appropriate therefore to require that $\eta$ be no smaller than some minimum cutoff: $\eta \geq \eta_{\min }$. In other words, the model waveform error need never be made smaller than

$$
\left\langle\delta h_{m} \mid \delta h_{m}\right\rangle \geq \eta_{\min }^{2}\left\langle\delta h_{R} \mid \delta h_{R}\right\rangle .
$$

This inequality can also be expressed as a condition on the signal-weighted amplitude and phase errors defined in Sec. II

$$
\overline{\delta \chi}_{m}^{2}+\overline{\delta \Phi}_{m}^{2} \geq \eta_{\min }^{2}\left({\overline{\delta \chi_{R}}}_{R}+\overline{\delta \Phi}_{R}^{2}\right) .
$$

These lower limits, Eqs. (47) or (48), on the model waveform error must be imposed simultaneously with the appropriate upper limits derived in Sec. II for measurement, Eq. (9), or detection, Eq. (16). The calibration error will interfere with the operation of a detector whenever it is impossible to satisfy the ideal-detector accuracy requirements of Sec. II and the calibration-error lower limits simultaneously.

To determine the appropriate value for $\eta_{\min }$, we note from Eq. (46) that a model waveform having the minimal error $\eta=\eta_{\min }$ could have an effect on the measured signal-to-noise ratio that is as large as

$$
\rho_{m} \geq \rho-\frac{1}{2 \rho}\left(1+\eta_{\min }\right)^{2}\left\langle\delta h_{R} \mid \delta h_{R}\right\rangle .
$$

Setting $\eta_{\min }=1$ corresponds to one natural choice: making the model waveform error greater than or equal to the calibration error. However, we see from Eq. (49) that the signal-to-noise ratio may be degraded by up to 4 times the effect of the calibration error alone in this case. So this obvious choice for $\eta_{\min }$ is probably too large. 
Given the relatively low cost of producing improved model waveforms (compared to the cost of improving the hardware needed to reduce the calibration error), it makes sense to adopt a stricter standard for $\eta_{\min }$. Another natural choice is to require that $\eta_{\min }$ be small enough to ensure that the most accurate model waveforms have no larger effect on the signal-to-noise ratio than the calibration error alone. From Eq. (49) we see that this requires $\left(1+\eta_{\min }\right)^{2} \leq 2$ or equivalently $\eta_{\min } \lesssim 0.4$. Reducing $\eta_{\min }$ below this value has little effect on the signal-to-noise ratio; while quickly increasing the computational cost of the most accurate model waveforms. So this choice should be close to optimal.

The most recent publicly available calibration data from Initial LIGO (S4), see Figs. 23 and 24 of Ref. [35], has the following limits on the frequency-domain calibration error: $0.03 \lessgtr \sqrt{\delta \chi_{R}^{2}+\delta \Phi_{R}^{2}} \lesssim 0.09$ for the L1 detector and $0.06 \leqq \sqrt{\delta \chi_{R}^{2}+\delta \Phi_{R}^{2}} \leqq 0.12$ for the $\mathrm{H} 1$ detector. Therefore the appropriate minimum error requirement, including the effects of the calibration error from Eq. (48), for Initial LIGO is ${ }^{4}$

$$
\begin{aligned}
& \sqrt{\overline{\delta \chi_{m}^{2}+\overline{\delta \Phi}_{m}^{2}}} \geq \eta_{\min } \sqrt{\left(\min \left|\delta \chi_{R}\right|\right)^{2}+\left(\min \left|\delta \Phi_{R}\right|\right)^{2}} \\
& \geq 0.012 .
\end{aligned}
$$

Model waveform error smaller than this limit will always be dominated by the calibration error. The magnitude of the calibration error in Initial LIGO is small enough that the calibration requirement, Eq. (50), is consistent with our basic measurement requirements, Eq. (9) or (32), for all but the very strongest sources: $\rho \gtrsim 80$. Therefore the presence of the calibration error will not affect the ideal-detector waveform accuracy standards for LIGO measurements, unless an extremely strong source is detected. This is not to say that the presence of the calibration error will have no effect on the accuracy of measurements for weaker sources; but the question of exactly how large those calibration-error effects are in that case must be decided by a somewhat more detailed analysis than the one presented here.

The lower limit on the model waveform error that arises from the presence of the calibration error is always consistent with the condition on the waveform error needed for detection, Eq. (16). From Eq. (49), the fractional change in the signal-to-noise ratio caused by a calibration error is

$$
\left|\frac{\delta \rho}{\rho}\right| \leq\left(1+\eta_{\min }\right)^{2} \frac{\left\langle\delta h_{R} \mid \delta h_{R}\right\rangle}{2 \rho^{2}} .
$$

For Initial LIGO $\left\langle\delta h_{R} \mid \delta h_{R}\right\rangle \lesssim 0.014$ (from the calibrationerror measurements quoted above), thus the signal-to-noise

\footnotetext{
${ }^{4}$ Although the calibration of the final data from Initial LIGO (S5) will be different from that of S4, the accuracy of the calibration is expected to be similar.
}

ratio $\rho$ would have to be smaller than about 1.7 (which is below any reasonable statistical threshold for detection) before the right side of Eq. (51) exceeds the detection limit $\epsilon_{\max }=0.005$. Thus, the current level of LIGO calibration error is never likely to influence the waveform accuracy requirements established in Sec. II B for detection.

\section{DISCUSSION}

In this paper we have developed a set of accuracy standards for model gravitational waveforms. These standards are designed to ensure that model waveforms are accurate enough to support the parameter measurement and detection needs of the gravitational wave data analysis community-without compromising the scientific content of the data, and without placing needless demands for accuracy on the waveform modeling community. In Sec. II we developed an optimal requirement for measurement purposes in Eq. (9) and for detection purposes in Eq. (16). These optimal standards place limits on the signal averaged amplitude and phase errors, $\overline{\delta \chi}$ and $\overline{\delta \Phi}$, respectively, as defined in Eqs. (7) and (8). The first row of Table I summarizes these accuracy standards (assuming the amplitude and phase errors to be comparable): $\rho$ represents the standard signal-to-noise ratio of the waveform, and $\epsilon_{\max }$ represents the maximum signal-to-noise mismatch tolerated by a given detection procedure.

Also listed in Table I are summaries of several sufficient conditions on the waveform accuracy developed in Sec. II C and described in detail in Eqs. (18), (19), (23), (24), and (30)-(33). The table entries for phase error assume that the amplitude and phase errors are comparable. These sufficient conditions are somewhat stronger than needed, but if satisfied they ensure the optimal requirements are satisfied as well. They are much simpler to apply. The quantities $\bar{C}$ and $C$, which appear in some of these conditions and are defined in Eqs. (22) and (27), compare different signal-to-noise measures of the waveforms. These quantities, which are waveform and detector noise dependent, are illustrated for nonspinning equalmass binary black-hole waveforms in Figs. 4 and 5 for the LIGO and LISA detectors, respectively.

TABLE I. Summary of model waveform accuracy requirements for various waveform error diagnostics for measurement purposes (column three) and for detection purposes (column four).

\begin{tabular}{lccc}
\hline \hline $\begin{array}{l}\text { Waveform Error } \\
\text { Diagnostic }\end{array}$ & $\begin{array}{c}\text { Equation } \\
\text { Numbers }\end{array}$ & $\begin{array}{c}\text { Measurement } \\
\text { Requirement }\end{array}$ & $\begin{array}{c}\text { Detection } \\
\text { Requirement }\end{array}$ \\
\hline$\overline{\delta \Phi}$ & (9) and (16) & $1 / \sqrt{2} \rho$ & $\sqrt{\epsilon_{\max }}$ \\
$\max |\delta \Phi|$ & $(18)$ and (19) & $1 / \sqrt{2} \rho$ & $\sqrt{\epsilon_{\max }}$ \\
$\max \left|A_{e} \delta \Phi\right| / A_{e}\left(f_{\mathrm{c}}\right)$ & $(23)$ and $(24)$ & $\bar{C} / \sqrt{2} \rho$ & $\sqrt{\epsilon_{\max }} \bar{C}$ \\
$\left\|A_{e} \delta \Phi(f)\right\| /\left\|A_{e}(f)\right\|$ & $(30)$ and (31) & $C / \sqrt{2} \rho$ & $\sqrt{\epsilon_{\max }} C$ \\
$\|\delta h(t)\| /\left\|h_{e}(t)\right\|$ & $(32)$ and (33) & $C / \rho$ & $\sqrt{2 \epsilon_{\max }} C$ \\
\hline \hline
\end{tabular}




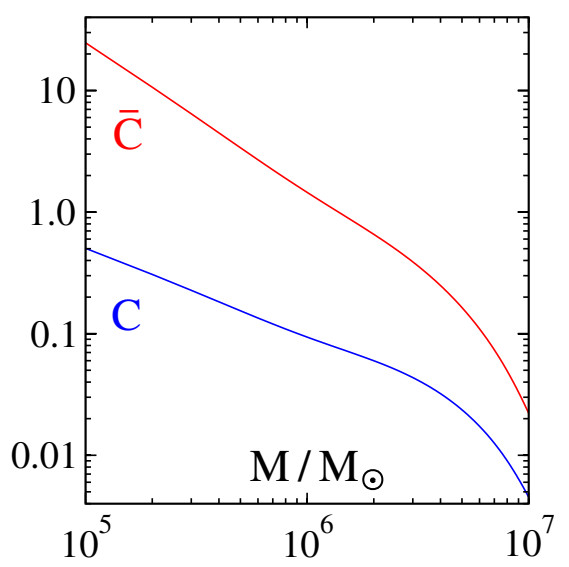

FIG. 5 (color online). Curves illustrate $\bar{C}$ and $C$, as defined in Eqs. (22) and (27), as functions of the total mass for nonspinning equal-mass binary black-hole waveforms using the Barack and Cutler [36] approximate LISA noise spectrum.

To apply these waveform accuracy standards to a particular detector, we must know the values of the parameters that appear in the requirements summarized in Table I. In particular we need to know $\max (\rho), \epsilon_{\max }, \min (\bar{C})$, and $\min (C)$ for that detector. The maximum signal-to-noise ratio, $\max (\rho)$, expected for Advanced LIGO is rather difficult to estimate, since the numbers and distribution of the relevant sources are not known yet. Here we need an estimate for $\max (\rho)$ only to compute the appropriate Advanced LIGO values of the requirements in Table I, so we take a rather simplistic approach: The threshold signalto-noise ratio for detecting compact binary inspirals currently used by Initial LIGO is about $\rho \approx 9$ (for $\mathrm{S} 4$, the most recent data published) [28]. Advanced LIGO is expected to be about 10 (or perhaps as much as 15) times more sensitive than Initial LIGO. Therefore, if no binary black-hole system is observed in the Initial LIGO (S5) data, then it is likely that the maximum signal-to-noise ratio for such events in the first year or two of Advanced LIGO observations will be no larger than about $\max (\rho) \approx$ 100. (This estimate might need to be increased or decreased by about $50 \%$ depending on the final sensitivity improvement of Advanced LIGO, and the final threshold adopted for detections in the S5 data; this final threshold will depend on the number of non-Gaussian artifacts found in the data.) Of course, if a binary black-hole signal is detected by Initial LIGO, then the expectation would be that a similar signal with 10 to 15 times that signal-to-noise ratio will be detected by Advanced LIGO. If Advanced LIGO template banks are constructed in the same way as those for Initial LIGO, then $\epsilon_{\max }=0.005$ is the appropriate mismatch tolerance for Advanced LIGO as well. We also need estimates of the quantities $\min (\bar{C})$ and $\min (C)$ for some of the waveform accuracy requirements. From Fig. 4 we see that the appropriate choices for these quantities for binary black-hole systems with masses in the range $5 \leq$ $M / M_{\odot} \leq 400$ for the Advanced LIGO detector are
TABLE II. Summary of model waveform accuracy requirements for the Advanced LIGO detector using various waveform error diagnostics for measurement purposes (column three) and for detection purposes (column four).

\begin{tabular}{lccc}
\hline \hline $\begin{array}{l}\text { Waveform Error } \\
\text { Diagnostic }\end{array}$ & $\begin{array}{c}\text { Equation } \\
\text { Numbers }\end{array}$ & $\begin{array}{c}\text { Measurement } \\
\text { Requirement }\end{array}$ & $\begin{array}{c}\text { Detection } \\
\text { Requirement }\end{array}$ \\
\hline$\overline{\overline{\delta \Phi}}$ & (9) and (16) & 0.007 & 0.07 \\
$\max |\delta \Phi|$ & $(18)$ and (19) & 0.007 & 0.07 \\
$\max \left|A_{e} \delta \Phi\right| / A_{e}\left(f_{\mathrm{c}}\right)$ & $(23)$ and (24) & 0.0004 & 0.004 \\
$\left\|A_{e} \delta \Phi(f)\right\| /\left\|A_{e}(f)\right\|$ & $(30)$ and (31) & 0.00014 & 0.0014 \\
$\|\delta h(t)\| /\left\|h_{e}(t)\right\|$ & $(32)$ and (33) & 0.0002 & 0.002 \\
\hline \hline
\end{tabular}

$\min (\bar{C}) \approx 0.06$ and $\min (C) \approx 0.02$. Using these parameter estimates, we summarize in Table II our current expectations for the model waveform accuracy standards that will be needed for Advanced LIGO data analysis.

The detection requirements listed in the first two rows of Table II should apply equally to Initial and to Advanced LIGO, since these requirements depend only on the parameter $\epsilon_{\max }$ which is determined by the properties of the search template bank and the accepted level of missed detections rather than the sensitivity of the detector. The somewhat stronger sufficient conditions that appear in rows $3-5$ of Table II also depend on the constants $\bar{C}$ and $C$, which are larger for the Initial LIGO case by factors of 10 and 2, respectively, because the appropriate mass range for Initial LIGO is smaller. The appropriate Initial LIGO requirements for measurement are less clear. The Initial LIGO detector is about 10 times less sensitive than the planned Advanced LIGO detector, so the expectation is that the accuracy requirements should be about 10 times weaker than those listed in Table II. However until a detection is actually made, no measurement requirements will be needed at all.

While the timetable and the technical specifications of the planned LISA detector are still being developed, we think it is appropriate to consider here what waveform accuracy standards this mission will eventually require from the waveform simulation community. The maximum signal-to-noise ratio for supermassive binary black-hole observations by LISA is expected to be much larger than the stellar mass black-hole observations made by LIGO. Supermassive black-hole mergers are expected to occur at a rate of about one merger per year within a sphere that extends to a cosmological redshift $z=2$ [37,38]. The largest signal-to-noise ratio for nonspinning equal-mass binaries located at $15 \mathrm{Gpc}$ (redshift $z \approx 2$ ) is about $\max (\rho) \approx 4 \times 10^{3}$ for an optimally oriented system with total mass $5 \times 10^{6} M_{\odot}$ [39]. Thus, the requirement on the accuracy of model waveforms for measurement purposes is likely to be much more demanding for LISA than for LIGO. If the LISA template banks are constructed in the same way as the LIGO template banks, then it is appropriate to assume the maximum mismatch parameter is 
TABLE III. Summary of model waveform accuracy requirements for the LISA detector for various waveform error diagnostics for measurement purposes (column three) and for detection purposes (column four).

\begin{tabular}{lccc}
\hline \hline $\begin{array}{l}\text { Waveform Error } \\
\text { Diagnostic }\end{array}$ & $\begin{array}{c}\text { Equation } \\
\text { Numbers }\end{array}$ & $\begin{array}{c}\text { Measurement } \\
\text { Requirement }\end{array}$ & $\begin{array}{c}\text { Detection } \\
\text { Requirement }\end{array}$ \\
\hline$\overline{\delta \Phi}$ & (9) and (16) & $2 \cdot 10^{-4}$ & 0.07 \\
$\max |\delta \Phi|$ & (18) and (19) & $2 \cdot 10^{-4}$ & 0.07 \\
$\max \left|A_{e} \delta \Phi\right| / A_{e}\left(f_{\mathrm{c}}\right)$ & $(23)$ and (24) & $4 \cdot 10^{-6}$ & 0.001 \\
$\left\|A_{e} \delta \Phi(f)\right\| /\left\|A_{e}(f)\right\|$ & $(30)$ and (31) & $7 \cdot 10^{-7}$ & 0.0003 \\
$\|\delta h(t)\| /\left\|h_{e}(t)\right\|$ & $(32)$ and (33) & $1 \cdot 10^{-6}$ & 0.0004 \\
\hline \hline
\end{tabular}

$\epsilon_{\max }=0.005$ as in the LIGO case. Figure 5 illustrates the quantities $\bar{C}$ and $C$ based on the approximate LISA noise curve constructed by Barack and Cutler [36] [cf. their Eq. (30)] using a $10^{-4} \mathrm{~Hz}$ low frequency cutoff. It follows that $\min (\bar{C}) \approx 0.02$ and $\min (C) \approx 0.004$ for binary systems with total masses in the range $10^{5}<M / M_{\odot}<10^{7}$. Using these parameter estimates, we summarize in Table III our current expectations for the model waveform accuracy standards that will be needed for LISA data analysis.

The accuracy requirements for measurement and detection that we discuss here provide upper limits on the allowed errors of model waveforms. These upper limits are the only requirements on model waveform accuracy when the detector is ideal, i.e., when the response function of the detector is known with absolute precision. In Sec. III we discuss the additional requirements that must be imposed when the response function has an error. We show that the model waveform error need never be decreased to a level below a certain fraction, $\eta_{\min } \approx 0.4$, of the response function error. For Initial LIGO the calibration error is small enough that it will not affect the ability of the instrument to make detections at all. The effect of this error on the ability of Initial LIGO to make measurements is more complicated: The calibration error will degrade the quality of measurements made on sources with large signal-to-noise ratios, $\rho \gtrsim 80$, and decreasing the model waveform error below $\eta_{\min }$ times the response function error will not improve these measurements substantially. For weaker signals, $\rho \lesssim 80$, the calibration error is likely to degrade the quality of measurements to some extent, but the ideal-detector model waveform accuracy standards should nevertheless be enforced in this case.

\section{ACKNOWLEDGMENTS}

We thank Emanuele Berti, Curt Cutler, and Michele Vallisneri for illuminating discussions on the subject of gravitational wave data analysis, and its particular application to LISA. We also thank Stephen Fairhurst, Kip Thorne, and Alan Weinstein for a number of useful comments on a preliminary draft of this paper. This research was supported in part by a grant to Caltech from the Sherman Fairchild Foundation, by NSF Grants No. DMS-0553302, PHY-0601459, and PHY-0652995 to Caltech, by NSF Grant No. PHY-0555628 to Penn State, and by the Penn State Center for Gravitational Wave Physics under NSF cooperative agreement PHY-0114375.
[1] B. Abbott et al. (LIGO Scientific Collaboration), arXiv:0711.3041.

[2] F. Acernese et al. (Virgo Collaboration), Classical Quantum Gravity 25, 114045 (2008).

[3] H. Grote (GEO600 Collaboration), Classical Quantum Gravity 25, 114043 (2008).

[4] M. Shibata and K. Uryu, Prog. Theor. Phys. 107, 265 (2002).

[5] F. Pretorius, Phys. Rev. Lett. 95, 121101 (2005).

[6] M. Campanelli, C.O. Lousto, P. Marronetti, and Y. Zlochower, Phys. Rev. Lett. 96, 111101 (2006).

[7] J.A. Faber, T.W. Baumgarte, S.L. Shapiro, and K. Taniguchi, Astrophys. J. 641, L93 (2006).

[8] J. G. Baker, J. Centrella, D.-I. Choi, M. Koppitz, and J. van Meter, Phys. Rev. Lett. 96, 111102 (2006).

[9] P. Diener, F. Herrmann, D. Pollney, E. Schnetter, E. Seidel, R. Takahashi, J. Thornburg, and J. Ventrella, Phys. Rev. Lett. 96, 121101 (2006).

[10] F. Loffler, L. Rezzolla, and M. Ansorg, Phys. Rev. D 74, 104018 (2006).

[11] F. Herrmann, I. Hinder, D. Shoemaker, and P. Laguna,
Classical Quantum Gravity 24, S33 (2007).

[12] B. Brügmann, J. A. González, M. Hannam, S. Husa, U. Sperhake, and W. Tichy, Phys. Rev. D 77, 024027 (2008).

[13] M. A. Scheel, M. Boyle, T. Chu, L.E. Kidder, K. D. Matthews, and H.P. Pfeiffer, arXiv:0810.1767.

[14] M. A. Miller, Phys. Rev. D 71, 104016 (2005).

[15] S. Fairhurst, "Required Waveform Accuracy" (unpublished).

[16] S. Anderson et al., Report No. LIGO-T010095-00-Z, 2001.

[17] M. Boyle, D. A. Brown, L. Pekowsky, H. P. Pfeiffer, and M. A. Scheel (unpublished).

[18] B. Abbott et al. (LIGO Scientific), Phys. Rev. D 69, 082004 (2004).

[19] P. Jaranowski and A. Krolak, Phys. Rev. D 59, 063003 (1999).

[20] R. J. Dupuis and G. Woan, Phys. Rev. D 72, 102002 (2005).

[21] L. S. Finn, Phys. Rev. D 46, 5236 (1992).

[22] L. S. Finn and D. F. Chernoff, Phys. Rev. D 47, 2198 (1993). 
[23] C. Cutler and E.E. Flanagan, Phys. Rev. D 49, 2658 (1994).

[24] B. J. Owen, Phys. Rev. D 53, 6749 (1996).

[25] B. Abbott et al. (LIGO Scientific Collaboration), Phys. Rev. D 69, 122001 (2004).

[26] B. Abbott et al. (LIGO Scientific Collaboration), Phys. Rev. D 72, 082001 (2005).

[27] B. Abbott et al. (LIGO Scientific Collaboration and TAMA Collaboration), Phys. Rev. D 73, 102002 (2006).

[28] B. Abbott et al. (LIGO Scientific Collaboration), Phys. Rev. D 77, 062002 (2008).

[29] T. A. Apostolatos, C. Cutler, G. J. Sussman, and K. S. Thorne, Phys. Rev. D 49, 6274 (1994).

[30] A. Lazzarini and R. Weiss, Report No. LIGO-E95001802-E, 1995; See also http://www.ligo.caltech.edu/ $\sim$ jzweizig/distribution/LSC_Data/.

[31] LIGO Scientific Collaboration, GWINC: Gravitational
Wave Interferometer Noise Calculator, http://lhocds.ligowa.caltech.edu:8000/advligo/GWINC.

[32] D. A. Brown, (for the LIGO Scientific Collaboration and Virgo Collaboration), Report No. LIGO-G080178-04-Z, 2008.

[33] B. Allen, Report No. LIGO-T960189-00-E, 1996.

[34] S. Bose (unpublished).

[35] A. Dietz, J. Garofoli, G. Gonzalez, M. Landry, B. O'Reilly, and M. Sung, Report No. LIGO-T050262-01D, 2006.

[36] L. Barack and C. Cutler, Phys. Rev. D 70, 122002 (2004).

[37] A. Sesana, F. Haardt, P. Madau, and M. Volonteri, Classical Quantum Gravity 22, S363 (2005).

[38] A. Sesana, F. Haardt, P. Madau, and M. Volonteri, Astrophys. J. 623, 23 (2005).

[39] A. Buonanno, G. B. Cook, and F. Pretorius, Phys. Rev. D 75, 124018 (2007). 